\begin{tabular}{l|l} 
REVISTA Revista Educación \\
EDUEACIÓN
\end{tabular}$\quad \begin{aligned} & \text { ISSN: 0379-7082 } \\
& \text { ISSN: 2215-2644 } \\
& \text { revedu@gmail.com } \\
& \text { Universidad de Costa Rica } \\
& \text { Costa Rica }\end{aligned}$

\title{
Teorías implícitas sobre inteligencia de niños escolarizados
}

\author{
Villamizar Acevedo, Gustavo \\ Teorías implícitas sobre inteligencia de niños escolarizados \\ Revista Educación, vol. 43, núm. 1, 2019 \\ Universidad de Costa Rica, Costa Rica \\ Disponible en: http://www.redalyc.org/articulo.oa?id=44057415002 \\ DOI: https://doi.org/10.15517/revedu.v43i1.27765
}

Esta obra está bajo una Licencia Creative Commons Atribución-NoComercial-SinDerivar 3.0 Internacional. 


\section{Teorías implícitas sobre inteligencia de niños escolarizados}

\author{
Implicit Theories About Intelligence of School Children \\ Gustavo Villamizar Acevedo 1 \\ Universidad Pontificia Bolivariana, Colombia \\ gustavo.villamizar@upb.edu.co
}

DOI: https://doi.org/10.15517/revedu.v43i1.27765

Redalyc: http://www.redalyc.org/articulo.oa?id=44057415002

Recepción: 15 Marzo 2017

Aprobación: 24 Noviembre 2018

\section{Resumen:}

Tomando como base la existencia de dos teorías personales sobre la inteligencia, la fija y la modificable, se realizó una investigación descriptiva con el objetivo de identificar las teorías implícitas de un grupo de niños y niñas sobre inteligencia. Para identificar dichas teorías se aplicó la Escala Teorías Implícitas sobre la Inteligencia, a 220 menores escolarizados, 157 de género femenino y 63 masculinos, con una media de edad de 11.53 años de edad, con una desviación estándar de .568. Para los datos se trabajó básicamente con medidas. Los resultados mostraron predominancia de las teorías de crecimiento, valoración del reconocimiento de los otros, así como de las tareas realizadas y asociación entre inteligencia y aprendizaje.

Palabras ClaVE: teorías, teorías implícitas, inteligencia, teoría fija, teoría modificable.

\section{Abstract:}

On the basis of the existence of two personal theories about intelligence, the fixed and the modifiable, a descriptive investigation with the aim of identifying implicit intelligence theories was held with a group of children. To identify such theories, a scale about implicit theories was applied to 220 school children, 157 females and 63 males, with an average age of 11.53 years old and a standard deviation of 568 .Data was basically worked with measurements. The results showed predominance of the theories about growth, the personal recognition of others, as well as the tasks performed and the relationship between intelligence and learning.

KEYWORDS: theories, implicit theories, intelligence, fixed theory, modifiable theory.

\section{INTRODUCCIÓN}

La presente investigación tiene como objetivo identificar las teorías implícitas de un grupo de niñas y niños sobre inteligencia y se encuentra fundamentada teóricamente en el supuesto que la creación de teorías en búsqueda de explicar y comprender diversos fenómenos del mundo no es propiedad exclusiva de los científicos, sino que todos los seres humanos pueden hacerlo (Aparicio \& Hoyos, 2008), no siendo los niños y las niñas la excepción en este proceso de construcción. Estas creaciones humanas reciben diversos apelativos, como concepciones, creencias, teorías ingenuas, para efectos de esta investigación se asumió la denominación de teorías implícitas.

Las teorías implícitas según Jodelet (como se citó en Rodríguez \& González, 1995, p. 222) "son una forma de conocimiento específico que orienta la comunicación y comprensión del medio social, material e ideal”. Estas teorías dirigen gran parte del accionar humano y dar cuenta de ellas permite inferir cómo las personas se autoperciben y perciben a quienes le rodean (Faria, 2007).

Cuando las teorías implícitas se encuentran relacionadas con inteligencia Cabezas y Carpintero (2006, p. 133) las definen como el conjunto de "creencias que las personas elaboran sobre qué es la inteligencia y cuál es su naturaleza”. Para Goldstein (como se citó en Hall, 2005, p. 88) las teorías implícitas sobre inteligencia son las "ideas que las personas tienen sobre todo aquello que conforma la inteligencia".

\section{NotAS DE AUTOR}

1 Psicólogo de la Universidad Nacional de Colombia, Dr. en Educación de la Universidad Pedagógica Experimental Libertador (UPEL), miembro del Grupo de Investigación Análisis y Transformación Psicosocial Universidad Pontificia Bolivariana Bucaramanga 
Las teorías que las personas construyen sobre la inteligencia, juegan un papel importante, tanto a nivel individual como grupal. En el plano individual estas teorías permiten la construcción de un conjunto de explicaciones mentales plausibles, coherentes y en el plano social la construcción de una identidad social y personal gratificante, las cuales facilitan un mejor desempeño cognitivo y promueven el bienestar psicológico (Faria, 2002). Según Lammel y Guillén (2011, p. 18) "los individuos las utilizan en la vida cotidiana para emitir juicios sobre lo que debe ser considerado como inteligente".

Para estudiar las teorías personales sobre inteligencia se han establecido varios marcos conceptuales: las teorías implícitas de la inteligencia como fija o modificable (Dweck \& Leggett, 1988); las basadas en las representaciones sociales (Zubieta y Valencia, 2001) y las teorías implícitas de Legos vs. Expertos (Sternberg, 1985).

De los marcos conceptuales referenciados esta investigación se encuadra en la teoría propuesta por Dweck (2007) quien postula la existencia de dos teorías sobre inteligencia: la fija y la modificable. Esta teoría se encuentra relacionada con la de motivación por el logro y se estructuró a partir de investigaciones realizadas por Dweck y Leggett en 1988 (como se citó en Dweck, 2009).

Quien cree que la inteligencia es fija, de acuerdo con Dweck (2009) se concibe como un rasgo estable, no influido por el esfuerzo o la práctica, y sobre sí mismo piensa que "es como es", por tanto no va a cambiar, y aunque desee desempeñarse mejor y parecer inteligente, este tipo de persona optan por evitar los desafíos, pues considera que son difíciles y no tiene garantía de éxito, por lo tanto en vez de asumir el riesgo de fallar e impactar negativamente su autoimagen, se apega a lo que ya sabe. Tienden a darse fácilmente por vencidos, pues perciben los obstáculos como fuerzas externas que se ponen en su camino, por lo cual considera el esforzarse como ineficaz, llegando a pensar que la acción más inteligente que pueden hacer es evitar los desafíos lo más que se pueda.

Este tipo de persona llega a pensar que cualquier crítica a sus capacidades es una crítica personal y terminan ignorándolas, evitando los cambios. Usualmente, cuando otros tienen éxito, intenta convencerse a sí misma y a las personas a su alrededor, que ese éxito se debe a la suerte, logrando poner en duda sus propios resultados. Finalmente, como fruto de estos pensamientos, logran confirmar su visión determinista del mundo, y el hecho de que "es como es".

Carpintero, Cabezas, González y Fernández (2003), caracterizan a los que conciben la inteligencia fija, como personas que se preocupan por los resultados, creen que los problemas y errores son expresiones de poca inteligencia, que el esfuerzo riñe con la inteligencia y que evitan situaciones que generen información negativa sobre sí.

En el campo escolar quienes conciben la inteligencia como fija, según Stocker y Faria (2012) se preocupan más por responder a las demandas de la escuela y en realizar las tareas que en desarrollar sus capacidades intelectuales, pues creen que su inteligencia está determinada. Al respecto Redondo, Inglés y García-Fernández (2014, p. 483) afirman que estudiantes con estas concepciones, desde el punto de vista motivacional "se orientan extrínsecamente puesto que su objetivo principal es obtener juicios positivos de los demás”.

Las personas quienes piensan que la inteligencia es modificable, se caracterizan según Binder (como se citó en Gayón, 2010) por creer que a través del esfuerzo y la práctica la inteligencia puede mejorarse, a este tipo de personas les gusta enfrentar retos y tareas difíciles, valoran positivamente el esfuerzo, la crítica y el éxito ajeno, además de ello los obstáculos los incentivan, los perciben como parte del éxito, ven el fracaso como una oportunidad para aprender, consideran necesario el esfuerzo para crecer y demostrar las competencias que poseen. No toman la crítica a nivel personal, se asume como una crítica a sus habilidades, la cual pueden cambiar y mejorar. El éxito de otras personas sirve de inspirador, y genera una reacción positiva que lo motiva a mantener el aprendizaje y mejorar. Este tipo de personas creen que su evolución intelectual se alcanza a través del compromiso, el esfuerzo y la inversión personal (Stocker \& Faria ,2012). En el plano 
motivacional "tiende a orientarse más intrínsecamente puesto que su objetivo es aumentar sus capacidades y habilidades” (Redondo, Inglés \& García-Fernández (2014, p. 483).

Carpintero, Cabezas, González y Fernández (2003) describen a los que conciben la inteligencia como modificable de la siguiente forma: personas preocupadas por el proceso antes que por los resultados, ven los problemas y errores como retos, valoran el esfuerzo para extender las capacidades cognitivas y buscan interactuar con quienes le permitan incrementar su conocimiento.

Diversas investigaciones se han realizado sobre esta temática, Cheng, Hau, Wen, y Kong (2000) estudiando a 1.650 estudiantes chinos de diferentes edades y niveles educativos, detectaron que a medida que se incrementa la edad aumenta el predominio de la creencia de la inteligencia como algo fijo. Boruchovitch (2001) encontró en un grupo de estudiantes brasileños los cuales a pesar de valorar el esfuerzo para alcanzar buen rendimiento académico positivamente, consideran la inteligencia como algo estable. Faria (2002) halló en infantes menores de 11 años predominio de la teoría modificable, pero también evidenció que a medida que los niños incrementan su escolaridad dicha creencia pierde valor. Vezeau y Bouffard (2002), encontraron en un grupo de niños franceses de primer nivel de secundaria mayor preeminencia de la teoría fija que los de tercer nivel.

Carpintero, Cabezas, González y Poveda (2003) hallaron en un grupo de niños y niñas españoles de Educación Primaria predominio de la concepción modificable. En un estudio realizado con escolares mexicanos de primaria, González (2003) halló predominancia de las teorías estáticas. Faria, Pepi y Alesi (2006) encontraron en estudiantes italianos preferencia por las teorías modificables. Massa (2013) encontró en menores italianos que se encontraban cursando educación primaria predominancia de las teorías modificables de la inteligencia.

Ciochină y Faria (2006) al realizar un estudio comparado entre estudiantes portugueses y rumanos notaron mayor predominio en los portugueses por la teoría fija que en los rumanos. Couceiro y Cunha (2012) encontraron en niños portugueses predominancia por la teoría modificable.

Perret, Dumesny, Grandjean y Muonghane (2011) realizaron un estudio donde compararon las teorías implícitas sobre inteligencia entre niños con trastornos de aprendizaje y sin ellos, encontrando que en los niños y las niñas sin problemas de aprendizaje, al contrario de quienes sí los tienen, predominan las teorías modificables.

Souchal y Toczek (2010) compararon las teorías de menores de dos grupos socioeconómicos diferentes notando en los pertenecientes a las clases desfavorecidas predominio de la teoría fija, situación opuesta a los más favorecidos económicamente que mostraron predilección por la teoría modificable.

Entre los estudios realizados en Colombia, Gayón (2010) encontró en un grupo de estudiantes de sexto grado de Barrancabermeja (Santander-Colombia) predominancia de las teorías fijas y correlación entre las teorías implícitas expresadas por los progenitores y sus descendientes. Aparicio, Cala y Adarve (2010) detectaron en estudiantes de V, VII y VII de un colegio privado del Municipio de Girón (SantanderColombia), prevalencia de la mentalidad de crecimiento y que dicha creencia se incrementa con la edad.

También se han realizado otro tipo de estudios buscando identificar las creencias sobre inteligencia independientes de la teoría de Dweck, como los expuestos por Leal-Sarabia, Reyes-Gómez, Méndez-Puga (2014), quienes compararon las concepciones de menores ubicados en zonas rurales y urbanas de Michoacán (México), las investigadoras detectaron la existencia de pocas diferencias en sus creencias respecto a la inteligencia, que la relacionan con los siguientes verbos: trabajar, aprender, estudiar y saber. En esas respuestas se evidencia la influencia de factores escolares.

La anterior investigación surgió a partir del trabajo de Lammel y Guillén (2011), quienes compararon las concepciones implícitas de la inteligencia de niños y niñas naturales de México y Francia. Ambos grupos creen que el ser humano nace con una inteligencia, pero para los mexicanos se transmiten genéticamente, los menores no son más inteligentes que sus padres, y piensan que todos los miembros de una familia tienen igual nivel intelectual. Los franceses creen que la inteligencia depende de cada persona, que no se transfiere por 
herencia y no es idéntica entre los diferentes miembros de la familia. En cuanto a la relación entre inteligencia y desarrollo, los mexicanos opinan que la inteligencia aumenta con la edad, por tanto los más viejos son más inteligentes. Los franceses estiman que la inteligencia llega a la cúspide en la adultez y disminuye con la edad. Los integrantes de ambos grupos creen que la población es más inteligente que las de antes.

Musci (2010) al indagar sobre las concepciones infantiles sobre inteligencia, identificó la existencia de un prototipo de vinculado "con el éxito a la hora de cumplir con las demandas escolares" (p. 410). GonzálezLeandro, Pelechado y García (2008) entrevistaron un grupo de adolescentes sobre lo que pensaban respecto a cómo son las personas inteligentes, ellos manifestaron que deben ser más geniales que las otras, poseer alta capacidad de asombro, pueden afrontar la incertidumbre adecuadamente, con alta capacidad para resolver problemas, y con buen sentido del humor. García (2005) detectó en un grupo de estudiantes de secundaria mexicanos la creencia de que la inteligencia es una capacidad innata, inmutable, inflexible y cuantificable.

Las investigaciones referenciadas no dejan ver predominio de una teoría sobre la otra, pero se evidencia que las teorías se van modificando a medida que las personas suben de edad y de nivel académico, y que dicho cambio va en detrimento de la teoría modificable de la inteligencia.

Identificar cuál de las teorías predomina es importante en el plano educativo, bajo la consideración que promover la teoría modificable tiende a disminuir el riesgo de deserción escolar e incrementar el rendimiento académico (Magno, 2012; Renaud-Dubé, Guay, Talbot, Taylor \& Koestner, 2015). El cambio de actitud frente a sus capacidades, es decir, modificar la creencia de que la inteligencia es una entidad fija, lleva al estudiantado, según Moore y Shaughnessy (2012) enfrentar y resolver problemas que en otros momentos hubiera evitado.

A partir de los resultados de las investigaciones reseñadas, se plantea la siguiente pregunta: ¿Cuáles son las teorías implícitas sobre inteligencia predominantes en niñas y niños escolarizados, la inteligencia como entidad fija o como entidad modificable?

De esta pregunta emerge la siguiente hipótesis: niñas y niños escolarizados que estudian en las Escuelas Normales de Bucaramanga y Piedecuesta conciben la inteligencia como una entidad fija.

\section{MÉTodo}

\section{Participantes}

Se trabajó con 220 estudiantes de dos instituciones oficiales pertenecientes al Área Metropolitana de la Ciudad de Bucaramanga, las Escuelas Normales de Bucaramanga y Piedecuesta, 157 mujeres y 63 hombres. La edad media de las mujeres es de 11.56 años con una desviación estándar de .535, la de los hombres es 11.45 con una desviación estándar de .638. La edad media del grupo total es 11.53 años de edad con una desviación estándar de .568. En lo atinente al nivel escolar 118 cursan el Grado V y 102 el Grado VI.

El nivel socioeconómico del grupo de estudiantes, tomando como criterio la clasificación dada por los estratos, los muestra viviendo en barrios de niveles socioeconómicos 2 y 3 , datos congruentes con la distribución social de los municipios donde se trabajó. Según informe de la Alcaldía de Piedecuesta el 92,74\% de las viviendas corresponden a los niveles socioeconómicos 2 y 3 (Becerra, 2012), en Bucaramanga el 45,7\% de las viviendas corresponden a esos niveles (Vanguardia Liberal, 2013).

\section{Instrumento}

Escala sobre Teorías Implícitas sobre la Inteligencia, construida por Abd-El-Fattah y Yates (2006), la cual fue traducida al español y adaptada para población del área metropolitana de Bucaramanga por la psicóloga Gayón (2010), quien aprobó el uso del instrumento para esta investigación. La escala consta de 14 ítems 
que miden las teorías implícitas sobre la inteligencia como fija o modificable, a cada una de estas categorías corresponden siete ítems. Para responder la escala hay cuatro opciones, totalmente de acuerdo, de acuerdo, en desacuerdo y totalmente en desacuerdo, las cuales para efecto de cuantificación recibieron los siguientes valores: 4, 3, 2 y 1 .

La confiabilidad de la obtenida por medio del alfa de Cronbach es de .76 para la subprueba sobre teoría de crecimiento y .83 para la relacionada con la teoría fija, estos datos muestran que la prueba es confiable. En lo relacionado con la validez de contenido, Gayón encontró que el instrumento mide los dos factores postulados por la teoría de Dweck (2007) modelo el cual fue confirmado por el análisis factorial. El cual también detectó la inexistencia de dificultades con los ítems.

Respecto al consentimiento informado, teniendo en cuenta que los participantes eran menores de edad, se elaboró un consentimiento dirigido a los padres y las madres con una solicitud expresa de aprobación por su parte para que sus hijos e hijas participaran en la investigación (Salkind, 1999). En este documento se les solicitó que lo respondiera uno de sus progenitores, también se les pidió que llenaran unos espacios correspondientes al nombre y estrato del barrio donde viven.

\section{Procedimiento}

Antes de iniciar la investigación se efectuó una reunión en cada una de las Direcciones de las Escuelas Normales, se les explicó el objetivo de esta y se les solicitó permiso para aplicar el instrumento. Una vez obtenido el permiso, se diálogo con docentes y con estudiantes, a estos últimos se les invitó a participar, a quienes accedieron se les informó que se requería el consentimiento de su madre o de su padre. Una vez obtenido el consentimiento se programó la fecha y lugar de aplicación de la prueba. Antes de responderla se le explicó al estudiantado cómo hacerlo. Los resultados fueron procesados en el paquete estadístico SPSS versión 19, una vez obtenidos, se analizaron y se comunicó al cuerpo docente.

\section{Resultados.}

Al tener en cuenta que la prueba utilizada evalúa las teorías fija y de crecimiento, planteadas por Dweck (2007), inicialmente se identificó la teoría por la cual el estudiantado manifestó sus preferencias, resultado que se muestra en la Tabla 1.

TABLA 1

Porcentaje de los y las estudiantes por teoría implícita de la inteligencia.

\begin{tabular}{ll}
\hline Teoría de Crecimiento & Teoría Fija \\
\hline $60,85 \%$ & $39,15 \%$ \\
\hline
\end{tabular}

Fuente: Elaboración propia.

Los datos encontrados dejan ver que los y las estudiantes se inclinan por considerar la inteligencia como una entidad modificable, en esa medida existe la tendencia a asumir como válida la teoría de crecimiento, la cual afirma que la inteligencia se puede incrementar como resultado del esfuerzo que cada persona realice. Estos resultados muestran la no comprobación de la hipótesis planteada.

Seguidamente se detallan los datos obtenidos en la subescala que mide inteligencia fija y se ejecuta un análisis de los ítems (Tabla 2). 
TABLA 2

Índice de porcentajes para la subescala Teoría Fija de la Inteligencia.

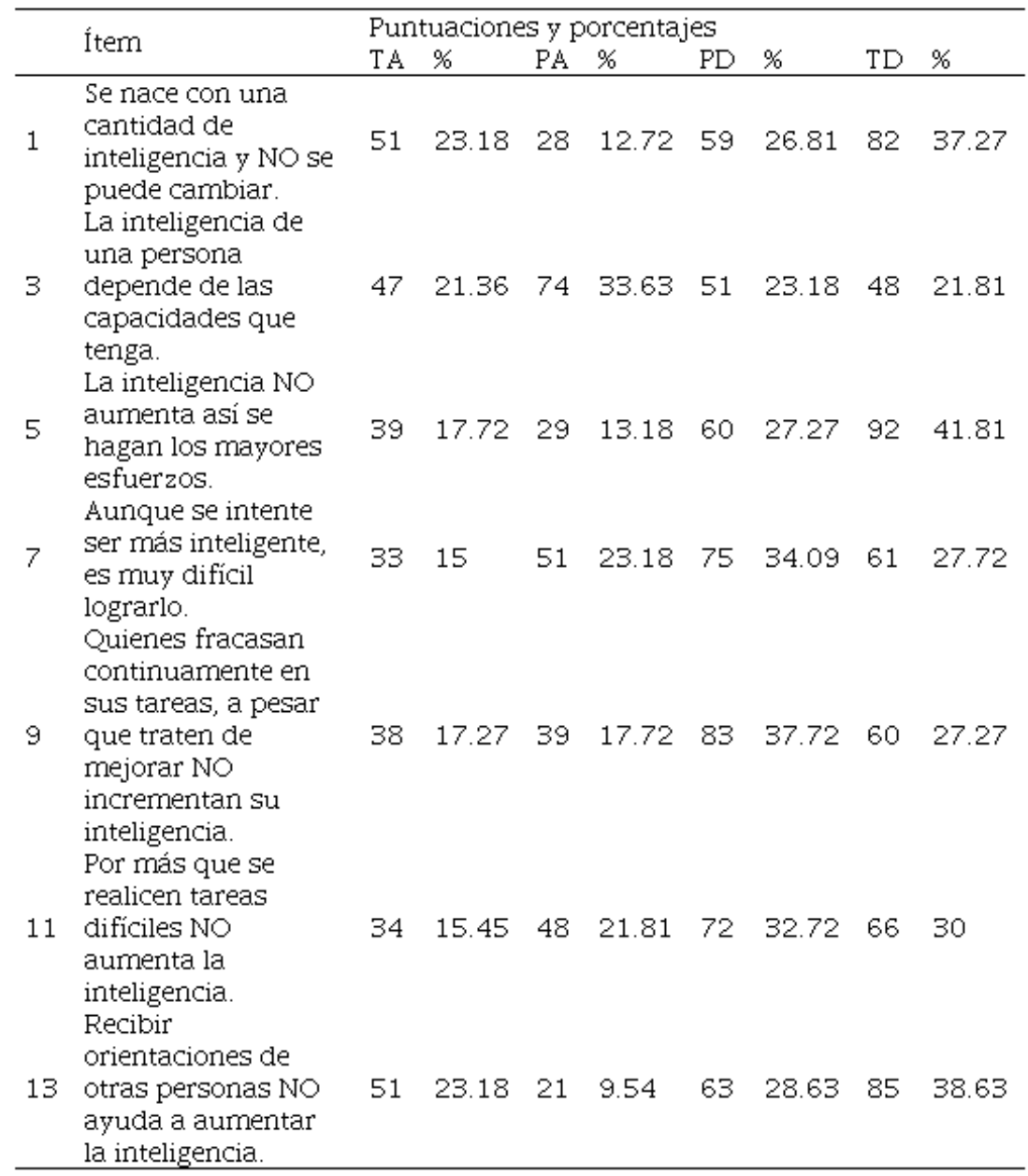

totalmente de acuerdo. PA: parcialmente de acuerdo. PD: parcialmente en desacuerdo. TD: Totalmente en desacuerdo. \%: porcentaje. Fuente: Elaboración propia.

\section{Análisis de ítems}

Los resultados del ítem 1 muestran que la mayoría de las personas encuestadas consideran que la inteligencia se puede cambiar, situación que deja ver predominio de la teoría de la inteligencia modificable.

Los datos del ítem 3 señalan que el 21,36\% de la muestra están totalmente de acuerdo y 33,63\% parcialmente de acuerdo con que el nivel intelectual de las personas se encuentra asociado con la capacidad que ella tenga, en esa medida la inteligencia se encuentra determinada por una condición inherente e independiente de factores externos, creencia que se enmarca en las teorías fijas de la inteligencia, yendo en contravía con la creencia identificada en el ítem anterior.

En el ítem 5 se indica que el $41,81 \%$ de las personas encuestadas están totalmente en desacuerdo con la creencia de que la inteligencia no es una condición fija, sino que puede incrementarse a partir del esfuerzo y del trabajo, y el $27,27 \%$ se encuentran parcialmente en desacuerdo con dicha creencia. Este puntaje deja entrever que la inteligencia es concebida como una entidad la cual se puede incrementar, fruto del trabajo y dedicación. 
Según los resultados del ítem 7, el 27,72\% de los escolares encuestados se encuentran totalmente en desacuerdo con la afirmación que dice que así se intente ser más inteligente, no es posible lograrlo y el 34,09\% están parcialmente en desacuerdo, el dato guarda correspondencia con el ítem anterior donde se evidencia la creencia en que la realización de ciertas actividades permite el desarrollo de la inteligencia.

Los datos arrojados por el ítem 9, evidencian que el 27,27\% de la muestra están totalmente de acuerdo y el $37,72 \%$ parcialmente de acuerdo con la creencia de que la inteligencia la puede incrementar cualquier persona, si intenta hacerlo, independientemente de su rendimiento escolar, esta posición muestra una creencia en la modificabilidad de la inteligencia, datos coinciden con lo detectado en las respuestas dadas en los dos anteriores ítems.

En lo atinente al reactivo 11, el 30\% de la muestra no está de acuerdo con la afirmación y el 32,72\% está parcialmente en desacuerdo, datos que permiten entrever la creencia en la modificabilidad de la inteligencia, bajo el supuesto que la inteligencia puede crecer en la medida que se realicen tareas y que entre más difíciles sean estas la posibilidad de incremento es mayor.

Respecto al ítem 13, del total de encuestados el 38,63\% están totalmente de acuerdo con la creencia de que es importante recibir orientaciones de otras personas para incrementar la inteligencia, y el 28,63\% parcialmente de acuerdo con ella, este hecho asociado con el anterior, que explica acerca del reconocimiento de las demás personas por las tareas que una persona realice, deja entrever el peso que se da a los demás en el desarrollo de la inteligencia.

En lo atinente a la creencia de la inteligencia como un proceso modificable, los resultados arrojados por la aplicación de la prueba se recogen en la Tabla 3. A continuación se presentan los resultados obtenidos en la subescala que mide creencia de la inteligencia modificable y se analizan los resultados.

TABLA 3

Índice de porcentajes para la subescala teoría modificable de la inteligencia.

\begin{tabular}{|c|c|c|c|c|c|c|c|c|c|}
\hline & & Punt & dacione: & $5 \mathrm{yp}$ & rcentaj & & & & \\
\hline & $11 \mathrm{EM}$ & TA & $\%$ & $\mathrm{PA}$ & $\%$ & $\mathrm{PD}$ & $\%$ & TD & $\%$ \\
\hline & Esforzarse por & & & & & & & & \\
\hline 2 & $\begin{array}{l}\text { hacer tareas } \\
\text { difíciles aumenta la } \\
\text { inteligencia. }\end{array}$ & 75 & 34.09 & 58 & 26.36 & 39 & 17.72 & 48 & 21.81 \\
\hline 4 & $\begin{array}{l}\text { Prepararse para } \\
\text { realizar las tareas } \\
\text { ayuda a aumentar } \\
\text { la inteligencia. }\end{array}$ & 85 & 38.63 & 46 & 20.9 & 54 & 24.54 & 35 & 15.9 \\
\hline 6 & $\begin{array}{l}\text { Si las personas se } \\
\text { lo proponen } \\
\text { aumentan su } \\
\text { inteligencia. }\end{array}$ & 97 & 44.09 & 42 & 19.09 & 26 & 11.81 & 55 & 25 \\
\hline 8 & $\begin{array}{l}\text { Hacer bien las } \\
\text { tareas aumenta la } \\
\text { inteligencia. }\end{array}$ & 66 & 30 & 73 & 33.18 & 36 & 16.36 & 45 & 20.45 \\
\hline & $\begin{array}{l}\text { El esforzarse por } \\
\text { hacer las cosas }\end{array}$ & & & & & & & & \\
\hline 10 & $\begin{array}{l}\text { mejor ayuda a } \\
\text { aumentar la } \\
\text { inteligencia. }\end{array}$ & 82 & 37.27 & 57 & 25.9 & 38 & 17.27 & 43 & 19.54 \\
\hline & $\begin{array}{l}\text { El reconocimiento } \\
\text { por las actividades }\end{array}$ & & & & & & & & \\
\hline 12 & $\begin{array}{l}\text { realizadas ayuda a } \\
\text { aumentar la. } \\
\text { inteligencia. }\end{array}$ & 82 & 37.27 & 60 & 27.27 & 36 & 16.36 & 42 & 19.09 \\
\hline 14 & $\begin{array}{l}\text { Cada vez que se } \\
\text { aprenden cosas } \\
\text { nuevas aumenta la } \\
\text { inteligencia. }\end{array}$ & 116 & 52.72 & 34 & 15.45 & 12 & 5.45 & 58 & 26.36 \\
\hline
\end{tabular}


totalmente de acuerdo. PA: parcialmente de acuerdo. PD: parcialmente n desacuerdo. TD: Totalmente en desacuerdo. \%: porcentaje. Fuente: Elaboración propia.

\section{Análisis de ítems}

En los datos del ítem 2 se nota que el 34,09\% de la muestra están totalmente de acuerdo con la afirmación del ítem y el 28,36\% parcialmente de acuerdo, lo cual supone que ellos creen que el esforzarse por realizar tareas difíciles contribuye al incremento de la inteligencia, desde esta perspectiva se puede suponer que para estas personas las tareas difíciles son vistas como retos cuya realización favorece el desarrollo intelectual. En estos datos se manifiesta una creencia en la teoría implícita de crecimiento y modificabilidad de la inteligencia.

Los datos del ítem 4 dejan ver que el 38,63\% de quienes respondieron la prueba están totalmente de acuerdo en que realizar tareas contribuye al incremento de la inteligencia, y el 20,9\% parcialmente de acuerdo con ello, situación que muestra una concepción de la inteligencia relacionada con la teoría de crecimiento.

De acuerdo con los datos del ítem 6, el 44,09\% de la muestra están totalmente de acuerdo con que es posible incrementar la inteligencia, y el 19,09\% parcialmente de acuerdo, con la anterior afirmación, pero para ello es necesario que las personas se lo propongan, es decir el incremento de la inteligencia está asociado a la realización de una serie de actividades y no se da como un factor biológico predeterminado. Estas respuestas muestran una creencia en las teorías implícitas de crecimiento.

Los resultados del ítem 8, muestran que el 30\% de los encuestados están totalmente de acuerdo en que la realización de las tareas aumenta la inteligencia, y el 33,18\% parcialmente de acuerdo, pero sí se hacen bien, es decir, no hacerlas por hacerlas, sino que es indispensable que ellas queden bien hechas. Este dato muestra predominio de la teoría implícita de crecimiento.

En cuanto al ítem 10, el 37,27\% de los encuestados se muestra totalmente de acuerdo en que el esforzarse por hacer las cosas bien posibilita el incremento de la inteligencia, y el 25,9\% parcialmente de acuerdo con ello, dato que evidencia la creencia en la teoría que plantea la modificabilidad e incremento de la inteligencia.

De acuerdo con los datos presentados en el ítem 12, el 37,27\% expresan estar totalmente de acuerdo con la afirmación y $27,27 \%$ parcialmente de acuerdo, estas respuestas desglosan, para quienes responden de esta manera, que la valoración y reconocimiento de los otros por las actividades realizadas les permite incrementar la inteligencia, bajo esta consideración la valoración de los otros es un factor motivacional externo importante para el desarrollo de las capacidades intelectuales.

Según los resultados del ítem 14, el 52,72\% de la muestra están totalmente de acuerdo con la creencia que el incremento de la inteligencia se encuentra asociado al aprendizaje sobre todo de cosas nuevas, y el 15,45\% están parcialmente de acuerdo con la aseveración, estas respuestas muestran aceptación de las teorías implícitas que hablan acerca de la modificabilidad e incremento de la inteligencia.

\section{Discusión}

Los resultados evidencian cómo un alto porcentaje de estudiantes se muestran de acuerdo en que la inteligencia es una capacidad que se puede incrementar como producto del esfuerzo, por realizar bien las tareas y demás actividades académicas. Estos resultados llevan a no acoger la hipótesis la cual planteaba que quienes participaban de la investigación asumían la inteligencia como fija.

Los resultados encontrados en esta investigación coinciden con los hallados por Faria (2002) y Carpintero, Cabezas, González y Poveda (2003) en menores españoles, y con los hallados por Aparicio, Cala y Adarve (2010) en una muestra de estudiantes de primaria en Girón Santander-Colombia), mas no así con los hallazgos de Gayón (2010) al trabajar con niños y niñas que viven en Barrancabermeja (Santander-Colombia) culturalmente más similares a los participantes de esta investigación. 
Es factible que estos resultados se encuentren asociados a factores de carácter social, la población estudiada por Gayón (2010) corresponde a un nivel socioeconómico de nivel 1, es decir, de familia económicamente desfavorecida, en cambio la abordada por esta investigación se encuentra en niveles 2 y 3 . Las diferencias a nivel económico entre estas familias, es significativa, las familias ubicadas en el nivel 1 no reciben lo correspondiente a un salario mínimo vigente, los que se encuentran en estrato 2 y 3 , perciben entre uno y tres salaros mínimos (Álzate, 2006).

Estos resultados coinciden con lo encontrado por Faria $(1996,2007)$ quien detectó la existencia de diferencias según nivel socioeconómico. Los niños y las niñas, del nivel medio, muestran tendencias a creer que la inteligencia es una entidad dinámica que se puede modificar. Souchal y Toczek (2010), también encontraron diferencias en las teorías sobre inteligencia según nivel social, imperando las modificables en el grupo de mayor nivel socioeconómico. El predominio de la teoría modificable de la inteligencia, en niños de mayor nivel socioeconómico, puede estar asociado con la creencia social que relaciona inteligencia con éxito económico, creencia que según Fandiño (2008) se trasfiere culturalmente.

La relevancia dada al esfuerzo deja entrever que si bien quienes participaron creen en el incremento de la capacidad intelectual, no ven esto como una resultante biológica, sino que se requiere del concurso de otras personas, es decir, se debe trabajar para conseguir incrementar la inteligencia. El incremento no se da sin razón alguna, responde a la realización de actividades, ejercicios y tareas que sean retadoras, es decir, que cuanto más difícil sea la tarea más posibilidades de incrementar la inteligencia. Al respecto Dweck y Leggett (como se citó en Escurra et ál., 2005) plantean que el estudiantado que valora el esfuerzo cree que ello es la causa de su éxito académico, la inteligencia es una entidad modificable para ellos y afrontan los problemas difíciles como retos.

En la valoración del esfuerzo se presenta coincidencia con lo encontrado por Boruchovitch (2001), con la salvedad que a diferencia de los menores brasileños la muestra en esta investigación considera la inteligencia como modificable.

Valorar el esfuerzo es de gran importancia para la creencia sobre el crecimiento de la inteligencia, pues permite inferir la no valoración de la suerte como variable interviniente en aspectos académicos. Esta creencia juega un papel positivo en la realización de programas de desarrollo intelectual, en la medida que existe la tendencia implícita a reconocer la importancia del trabajo intelectual en el desarrollo cognitivo.

Es significativo reconocer el valor dado al aprendizaje como factor posibilitador del crecimiento de la inteligencia, un 52,72\% de quienes participaron en la investigación consideran que aprender cosas nuevas incrementa la inteligencia, este dato puede estar asociado al alto valor que la sociedad le pone al aprendizaje. Por lo general, existe la tendencia a relacionar el estudio con el saber y al saber con la inteligencia, en esa medida cuanto más se aprenda más se sabe y por consiguiente más inteligente se es. Estos resultados coinciden con lo encontrado por Musci (2010), quien identificó la existencia de un prototipo de inteligencia vinculado con el éxito académico.

Respecto al papel que cumplen los otros en el proceso de incremento de la inteligencia, la muestra valora el reconocimiento recibido cuando se hacen bien las tareas y demás actividades académicas así como el apoyo que reciben para realizarlas. Esta apreciación se relaciona con lo encontrado por Faria (2005) quien notó que los adolescentes son muy sensibles a la retroalimentación sobre sus competencias personales, sobre todo cuando provienen del padre y de la madre o de sus compañeros y sus compañeras.

En términos generales es importante resaltar la creencia sobre la inteligencia como modificable, en la medida que ello, y de acuerdo a lo planteado por Gayón (2010), permite suponer cierto compromiso de la niñez con su desarrollo cognitivo, expresado a través de la realización de las obligaciones académicas que le permitan crecer intelectualmente. Estos resultados pueden ser altamente optimistas para el cuerpo docente, porque permiten suponer la existencia de un claro compromiso del personal de participantes en su proceso formativo y en su crecimiento personal, según lo planteado por Dweck (2007). 


\section{Conclusiones}

La fundamentación teórica de esta investigación permite suponer que las creencias construidas alrededor de la inteligencia juegan un papel de gran importancia en su desarrollo, y por tanto es una variable a considerar en los programas que propende por el incremento de este proceso cognitivo. Por tanto, se considera que la escuela debe conocer las creencias construidas tanto por el estudiantado como por el cuerpo docente, en la medida que el proceso de enseñanza y aprendizaje pueda estar influido por ellas.

Quienes participaron han considerado que la inteligencia se puede incrementar como producto del esfuerzo personal así como por la valoración y el apoyo de otras personas en la realización de actividades y tareas, este es un conocimiento de alto valor para el proceso de enseñanza aprendizaje, el cual debe llevar al profesorado a diseñar actividades de aprendizaje que impliquen a nivel individual esfuerzo y compromiso y a nivel grupal trabajo colaborativo y a los estudiantes a comprometerse con su evolución.

La tendencia a relacionar inteligencia con aprendizaje, presente en un alto número de quienes participaron, puede generar efectos contraproducentes, si se confunde inteligencia con rendimiento académico, asociación la cual no se presenta en este grupo, pues tiene una idea de aprendizaje más dinámica, asociada con conocer cosas nuevas, esto -según las creencias de la muestra- incrementa su inteligencia. Relacionar aprendizaje con rendimiento académico, medido este último con notas, puede generar un efecto paralizante, y llevar a la creencia de la inteligencia como fija, pues quienes no logran buenas notas, que por lo general son considerados poco inteligentes, no se esfuercen por alcanzar mejores resultados.

A pesar de la predominancia de las teorías implícitas de crecimiento, existe entre un buen número de ellos la creencia de que la inteligencia se encuentra asociada con factores de carácter genético, el 23,18\% está totalmente de acuerdo con esta afirmación y el 12,72\% parcialmente de acuerdo, lo que quiere decir que un alto porcentaje de estudiantes consideran que las capacidades intelectuales de las personas dependen de la herencia, están dadas al momento de nacer.

La creencia de la inteligencia asociada a factores genéticos, en cerca del $36 \%$ de los y las participantes debe llevar a las personas a cargo del proceso de formación a seguir indagando en las teorías construidas sobre inteligencia y a diseñar estrategias que modifiquen dicha creencia, la cual puede ser un obstáculo en el proceso de desarrollo cognitivo de niños y niñas.

Los hallazgos de esta investigación llevan a considerar la posibilidad de generar programas de carácter educativo los cuales solidifiquen la creencia de la inteligencia como un proceso que, a partir del trabajo y el esfuerzo, se puede incrementar, que ella no está previamente determinada y que para su desarrollo se requiere compromiso y dedicación.

\section{REFERENCIAS}

Abd-El-Fattah, S. \& Yates, G. (2006). Implicit Theory of Intelligence Scale: Testing for factorial invariance and mean structure. Recuperado de http://www.aare.edu.au/06pap/abd06289.pdf

Álzate, M. (2006). La estratificación socioeconómica para el cobro de los servicios públicos domiciliarios en Colombia ¿Solidaridad o focalización? Bogotá: Naciones Unidas- CEPAL.

Aparicio, M.; Cala, M, \& Adarve, S. (2010). Teorías implícitas de la inteligencia en estudiantes de quinto a séptimo grado de un colegio del municipio de Girón, Santander, En II Congreso Internacional de Investigación y Práctica Profesional en Psicología (pp. 312-314). Buenos Aires, Argentina: Universidad de Buenos Aires.

Aparicio, J. \& Hoyos, O. (2008). Enseñanza para el cambio de las representaciones sobre el aprendizaje. Universitas Psychologica, 7 (3), 725-737.

Becerra, A. (2012). Plan de desarrollo 2012-2015. Alcaldía de Piedecuesta: Piedecuesta.

Boruchovitch, E. (2001). Conhecendo as crencas sobre inteligencia, esforco e sorte de alunos brasileiros em tarefas escolares. Psicología: Reflexao e Critica, 14, (3), 461-467. 
Cabezas, D. y Carpintero, E. (2006). Teorías implícitas sobre inteligencia en docentes. EduPykhé, 5 (1), 129-142.

Carpintero, E., Cabezas, D., González, C., \& Poveda, M. (2003). Análisis de las Teorías Implícitas de la Inteligencia en Alumnos de Educación Primaria. EduPykhé, 2 (1), 81-105.

Cheng, Z., Hau, K., Wen, J., \& Kong, C. (2000). Chinese Students' Implicit Theories of Intelligence and Other Personal Attributes: Cross-Domain Generality and Age-Related differences. Paper presented at the Annual Meeting of the American Educational Research Association. New Orleans.

Ciochină, L. \& Faria, L. (2006). Concepções pessoais de inteligência de estudantes portugueses e romenos: Estudo preliminar de análise factorial confirmatória. Psychologica, 41, 171-\#191.

Couceiro, A. \& Cunha, R. (2012). A trilogia atribuições causais, concepções pessoais de inteligência e mensagens de feedback: um contributo para a compreensão do fracasso escolar. Práxis educacional, 8 (13), 83-105.

Dweck, C. \& Leggett, E. (1988). A Social-Cognitive Approach to Motivation and Personality. Psychological Review, 95 (2), 256-273.

Dweck, C. (2007). La actitud del éxito. Bogotá: Vergara.

Dweck, C. (2009). Theories of Intelligence. Recuperado de www.education.com/ reference /article/theories-ofintelligence/

Escurra, M., Delgado, A., Guevara, G., Torres, M., Quezada, R., Morocho, J., Rivas, G. \& Santos, J. (2005). Relación entre autoconcepto de las competencias, las metas académicas en alumnos universitarios de la ciudad de Lima, Revista IIPSI, 8 (1), 87-106.

Fandiño, Y. (2008). Una enseñanza e investigación inteligentes de la inteligencia para el éxito escolar y el éxito en la vida cotidiana. Revista Iberoamericana de la Educación. 46 (9), 1-12.

Faria, L. (1996). Desenvolvimiento diferencial das concepcoes pessoais de inteligencia durante a adolescencia. Revista Portuguesa de Pedagogía, XXX (1), 17-33.

Faria, L. (2002). Teorías implícitas da inteligencia: Estudos no contexto escolar portugués. Revista da Vetor Editora, $8(1), 13-20$.

Faria, L. (2005). Desenvolvimento do auto-conceito físico nas crianças e nos adolescentes. Análise Psicológica, 23 (4), 361-371.

Faria, L., Pepi, A., \& Alesi, M. (2006). Personal conceptions of intelligence: Cross cultural comparisons between Portuguese and Italian students. Social Behavior and Personality, 7 (34), 815-826.

Faria, L. (2007). Concepções pessoais de inteligência: na senda de um modelo organizador e integrador no domínio da motivação. Revista de Psicologia da Vetor Editora, 8 (1), 13-20.

García, G. (2005). Las inteligencias múltiples en las escuelas secundarias. El caso de una institución pública del Estado de México, Tiempos de Educar, 6 (12), 289-315.

Gayón, L. (2010). Relación entre las teorías implícitas de la inteligencia de padres e hijos y rendimiento académico (Trabajo de grado), Universidad Pontificia Bolivariana, Bucaramanga.

González, L. (2003). Teorías implícitas sobre la inteligencia: su modificación mediante un programa de inteligencia práctica para la escuela primaria. Recuperado de http://www.cibernetia.com/tesis_es/PSICOLOGIA/ PSICOLOGIA_DEL_NI\%D1O_Y_DEL_ADOLESCENTE/PSICOLOGIA_ESCOLAR/1

González-Leandro, P., Pelechado, V., \& García, L. (2008). Concepción lega de sabio, inteligente y listo en adolescentes. International Journal of Clinical and Health Psychology, 8 (2), 495-508.

Hall, L. (2005). Dictionary of multicultural psychology: issues, terms and concepts. Recuperado de http:// knowledge.sagepub.com/view/dictionary-of-multicultural-psychology/ AGE.xml

Lammel, A., \& Guillén, E. (2011). Las concepciones implícitas de la inteligencia en contextos culturales diferentes (México y Francia). Revista Electrónica de Psicología Iztacala, 14(2), 15-33.

Leal-Sarabia, R., Reyes-Gómez, E., \& Méndez-Puga, A. (2014). La inteligencia vista desde la perspectiva de niños y niñas urbanos y rurales en el estado de Michoacán, Revista de Educación y Desarrollo, 28, 45-53.

Magno, C. (2012). Implicit Theories of Intelligence, Achievement Goal Orientation, and Academic Achievement of Engineering Students. The International Journal of Research and Review, 9, 32-43. 
Massa, S. (2013, junio). Lo sviluppo del pensiero critico nella scuola primaria: il ruolo delle credenze degli insegnanti. Ponencia presentada en el VII Seminario SIRD, Roma.

Moore, T. \& Shaughnessy, M. (2012). Carol Dweck's views on achievement and intelligence: implications for education. Research Journal in Organizational Psychology \& Educational Studies 1(3) 174-184.

Musci, M. (2010). Las concepciones infantiles sobre inteligencia. En II Congreso Internacional de Investigación y Práctica Profesional en Psicología (pp. 409-411). Buenos Aires, Argentina: Universidad de Buenos Aires.

Perret, P., Dumesny, M., Grandjean, D. \& Muonghane, V. (2011). Troubles des apprentissages et théories implicites de l'intelligence. Développements, 8, 35-43.

Redondo, J., Ingles, C. \& García-Fernández, J. (2014). Conducta prosocial y autoatribuciones académicas en Educación Secundaria Obligatoria. Anales de Psicología, 30 (2), 482-489.

Renaud-Dubé, A., Guay, F., Talbot, D., Taylor, G. \& Koestner, R. (2015). The relations between implicit intelligence beliefs, autonomous academic motivation, and school persistence intentions: a mediation model. Social Psychology of Education, 18, 1-18.

Rodríguez, A. \& González, R. (1995). Cinco hipótesis sobre las teorías implícitas. Revista de Psicología General y Aplicada, 48 (3), 221-229.

Salkind, N. (1999). Métodos de investigación. México D.F.: Prentice Hall.

Souchal, C. \& Toczek, M. (2010). Buts de réussite, conceptions de l'intelligence, différences de performances liées à l'appartenance socio-économique des élèves: de nouvelles hypothèses explicatives? Les Sciences de l'éducation Pour l'Ėre nouvelle, 43, 13-35.

Sternberg, R. (1985). Implicit theories of intelligence, creativity, and wisdom. Journal of Personality and Social Psychology, 49 (3), 607-627.

Stocker, J. \& Faria, L (2012). Competencia percebida no ensino secundário: do conceito à avaliação através de um questionário compósito. Psicologia, 26 (2), 113-140.

Vanguardia Liberal (2013, septiembre 30). Así se define el estrato de los predios en Bucaramanga. Recuperado de http://www.vanguardia.com/santander/bucaramanga/227315-asi-se-define-el-estrato-de-lospredios-en-bucaramanga

Vezeau, C. \& Bouffard, T. (2002). Relation entre la théorie implicite de l'intelligence et les buts d'apprentissage chez des élèves du secondaire. Revue des sciences de l'éducation, 28 (3), 675-692.

Zubieta, E. y Valencia, J. (2001). Representaciones sociales de la inteligencia. Psicodebate, 2, 169-196.

\title{
Apéndice A
}

\section{ESCALA SOBRE TEORÍAS IMPLÍCITAS SOBRE LA INTELIGENCIA}

\author{
Edad Curso \\ Colegio Ciudad
}

\begin{tabular}{|l|l|l|l|l|l|}
\hline$N^{\circ}$ & AFIRMACIÓN & TA & PA & PD & D \\
\hline 1 & $\begin{array}{l}\text { Demuestro que soy inteligente cuando hago las cosas } \\
\text { mejor que los demás. }\end{array}$ & $\mathrm{X}$ & & \\
\hline
\end{tabular}

TA: totalmente de acuerdo. PA: parcialmente de acuerdo. PD: parcialmente en desacuerdo. TD: totalmente en desacuerdo

Responder de esta forma muestra que estoy PARCIALMENTE DE ACUERDO con la afirmación, no lo estoy tanto como para estar TOTALMENTE DE ACUERDO con ella.

Para responder este cuestionario, no hay límite de tiempo, NO hay respuestas buenas ni malas. 


\section{RESPONDA TODAS LAS AFIRMACIONES. GRACIAS POR SU COLABORACIÓN.}

\begin{tabular}{|c|c|c|c|c|c|}
\hline$N^{\circ}$ & AFIRMACIÓN & TA & PA & $\mathrm{PD}$ & $\mathrm{D}$ \\
\hline 1 & $\begin{array}{l}\text { Se nace con una cantidad de inteligencia y No se puede } \\
\text { cambiar. }\end{array}$ & & & & \\
\hline 2 & $\begin{array}{l}\text { Esforzar'se por hacer tareas difíciles aumenta la } \\
\text { inteligencia. }\end{array}$ & & & & \\
\hline 3 & $\begin{array}{l}\text { La inteligencia de una persona depende de las } \\
\text { capacidades que tenga. }\end{array}$ & & & & \\
\hline 4 & $\begin{array}{l}\text { Prepararse para realizar las tareas ayuda a aumentar la } \\
\text { inteligencia. }\end{array}$ & & & & \\
\hline 5 & $\begin{array}{l}\text { La inteligencia NO aumenta asi se hagan los mayores } \\
\text { esfuerzos. }\end{array}$ & & & & \\
\hline 6 & $\begin{array}{l}\text { Si las personas se lo proponen aumentan su } \\
\text { inteligencia. }\end{array}$ & & & & \\
\hline 7 & $\begin{array}{l}\text { Aunque se intente ser más inteligente, es muy difícil } \\
\text { lograrlo. }\end{array}$ & & & & \\
\hline 8 & Hacer bien las tareas aumenta la inteligencia. & & & & \\
\hline 9 & $\begin{array}{l}\text { Quienes fracasan continuamente en sus tareas, a pesar } \\
\text { que traten de mejorar NO incrementan su inteligencia. }\end{array}$ & & & & \\
\hline 10 & $\begin{array}{l}\text { El esforzarse por hacer las cosas mejor ayuda a } \\
\text { aumentar la inteligencia. }\end{array}$ & & & & \\
\hline 11 & $\begin{array}{l}\text { Por más que se realicen tareas difíciles NO aumenta la } \\
\text { inteligencia. }\end{array}$ & & & & \\
\hline 12 & $\begin{array}{l}\text { El reconocimiento por las actividades realizadas ayuda } \\
\text { a aumentar la inteligencia. }\end{array}$ & & & & \\
\hline 13 & $\begin{array}{l}\text { Recibir orientaciones de otras personas NO ayuda a } \\
\text { aumentar la inteligencia. }\end{array}$ & & & & \\
\hline 14 & $\begin{array}{l}\text { Cada vez que se aprenden cosas nuevas aumenta la } \\
\text { inteligencia. }\end{array}$ & & & & \\
\hline
\end{tabular}

\section{BY-NC-ND}

